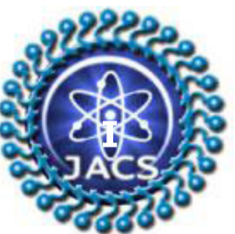

\title{
Indian \\ Journal of Advances in Chemical Science
}

Available online at

www.ijacskros.com

Indian Journal of Advances in Chemical Science S2 (2017) 1-5

\section{Enzyme Immobilization Methods and Role of Conductive Polymers in Fabrication of Enzymatic Biosensors}

\author{
N. Kumara Swamy*, S. Sandeep, A. S. Santhosh \\ Department of Chemistry, Sri Jayachamarajendra College of Engineering, Mysore - 570 006, Karnataka, India.
}

Received: 12 ${ }^{\text {th }}$ June 2017; Revised 29 $9^{\text {th }}$ July 2017; Accepted 04 ${ }^{\text {th }}$ August 2017

\begin{abstract}
Immobilization of enzymes on the transducer surface is a necessary and critical step in the design of biosensors. The conducting polymers play a very significant role in the development of enzymatic sensors having emerged as effective transducer materials. This paper briefly discusses various enzyme immobilization methods based on physical adsorption, covalent linking, entrapment, cross-linking, and also the role of conducting polymers as immobilization platforms in the fabrication of enzymatic biosensors. The study concluded that the choice of immobilization method and the conducting polymer greatly influence the enzyme orientation, loading, mobility, stability, structure, and biological activity which in turn affects the performance of biosensor in terms of sensitivity, selectivity, and stability.
\end{abstract}

Key words: Conducting polymers, Enzyme immobilization, Enzymatic sensors.

\section{INTRODUCTION}

Biosensor is an analytical device which specifically detects a particular analyte in a sample. A biosensor has three major components: (i) A biorecognition element or bioreceptor for the recognition of an analyte, (ii) an immobilization surface used for immobilization of biomolecule, and (iii) a transducer unit for conversion of biochemical reaction product into a recognizable signal [1]. Biosensors integrate biological recognition elements such as enzymes, antibodies, nucleic acids, and cells with electronic transducer equipped with an electronic amplifier (Figure 1). The majority of older-generation biosensors are based on one or more biomolecules used in conjunction with an electrode. The redox reaction could be detected electrochemically by measuring the loss or formation of substrate or product, respectively, by the use of a small mediator species that shuttles between the biomolecule and the electrode, or less commonly by direct electron transfer (ET) between the biomolecule redox site and the electrode [1,2]. However, direct ET can be difficult to achieve as the redox site of a biomolecule is often hidden deep inside the biomolecule. A recent trend in achieving direct ET involves modification of biomolecules or electrode surfaces through the use of novel conducting materials as mediators and design of functional biointerfaces. Thus, highly conductive organic transducers such as conductive polymers are gradually emerging as effective materials for the

*Corresponding Author:

E-mail: kumaryagati@gmail.com

Phone: +91-9741027970 development of next generation biosensor design for highly reliable, stable, and robust field-based diagnostic devices [1].

Conducting polymers mediate ET between the biorecognition layer and the final electrode. However, the major problem while fabricating enzyme-based biosensor is the immobilization of enzymes onto electrode matrix. Most of the time fabrication fails due to lose binding, chemical modification, denaturation, and loss of enzyme activity apart from instability of matrix [3]. These failures can be minimized or avoided through the proper deposition of conducting polymer matrix and also by adopting proper enzyme immobilization techniques. In the present paper, we discuss (i) the significance of conducting polymers in electrode fabrication and (ii) the methods involved in immobilization of enzymes.

\section{SIGNIFICANCE OF CONDUCTING} POLYMERS IN ELECTRODE FABRICATION Conducting polymers have attracted much interest as a suitable matrix for the entrapment of enzymes. They enhance speed, sensitivity, and versatility of biosensors in diagnostics to measure vital analytes. Electrically conducting polymers have considerable flexibility in the available chemical structure, which can be modified as required. Another advantage offered by conducting polymers is that they can be directly deposited on 


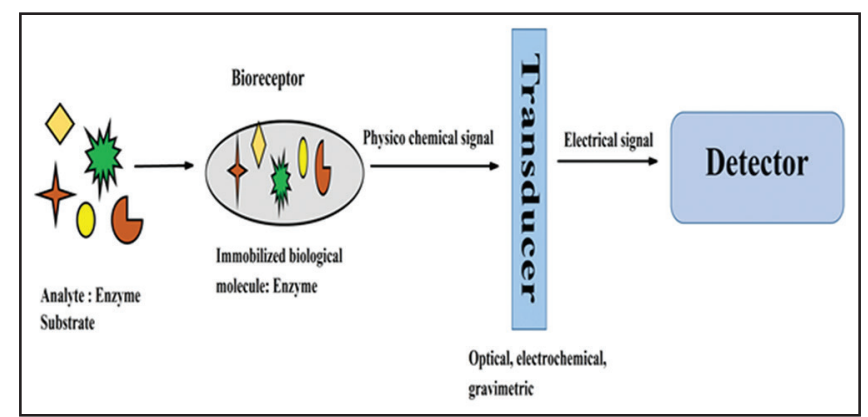

Figure 1: Scheme of a biosensor.

the electrode surface by electrochemical methods, while simultaneously trapping the protein molecules. Thus, it is possible to control the spatial distribution of the immobilized enzymes, the film thickness, and modulates the enzyme activity by changing the state of the polymer [3]. Conducting polymers are also known to be compatible with biological molecules in neutral aqueous solutions. They have the ability to efficiently transfer electric charge produced by the biochemical reaction to electronic circuit. Moreover, conducting polymers can be deposited over defined areas of electrodes. Numerous published papers reveal that the electro deposition of conducting polymers serves as good matrices for the immobilization of enzymes and they provide good detectability and fast response as the redox reaction of the substrate takes place in the bulk of the polymer layer [1].

\section{METHODSOFENZYMEIMMOBILIZATION} Enzyme immobilization is a very crucial step in the development of efficient biosensors with appropriate performances such as good operational and storage stability, high sensitivity, high selectivity, short response time, and high reproducibility. An immobilized enzyme has many operational advantages over free enzyme such as reusability, enhanced stability, continuous operational mode, rapid termination of reaction, easy separation of biocatalyst from product, and reduced cost of operation. Poor immobilization often leads to problems such as loss of enzyme, poor enzyme stability, and low shelf life of the biosensors [4]. To overcome these problems, several immobilization procedures have been developed, and the major schemes (Figure 2) are discussed below.

\subsection{Adsorption}

Enzyme adsorption onto solid supports is the easiest method of physical immobilization. Enzyme is dissolved in solution and the solid support is placed in contact with the enzyme solution for a fixed period of time. The unadsorbed enzyme is then removed by washing with buffer. The adsorption mechanisms are based on weak bonds such as Van der Waals forces and electrostatic and/or hydrophobic interactions [2]. Although this immobilization method causes little or no enzyme inactivation, it often experiences drawbacks such as loose binding of enzymes to the support and desorption of the enzyme. Thus, biosensors based on adsorbed enzyme suffer from non-specific adsorption of other proteins or substances, poor operational, and storage stability. Furthermore, since biomaterial is immobilized on the outer layer of conducting polymer, it gets leached out into sample solution during measurements. This decreases the lifetime stability of enzyme electrode [4].

\subsubsection{Physical adsorption}

Physical adsorption consists of simple deposition of an enzyme onto a surface and its attachment through weak bonds. This immobilization strategy has widely been used to develop enzymatic biosensors [2].

\subsubsection{Electrostatic interactions}

In this technique, enzymes are electrostatically immobilized onto charged support surface. When the isoelectric point of the enzyme is lower than the $\mathrm{pH}$ value of the solution, enzyme is negatively charged and it can bind to a positively charged support. Layerby-layer deposition and electrochemical doping techniques are based on electrostatic adsorption [2].

\subsubsection{Layer-by-layer deposition}

In layer-by-layer deposition technique, alternate layers of polyelectrolyte (polycation/polyanion) and enzyme with opposite charges are placed one above the other. Initially, an electrode has to be modified with a negative (or positive) charge which is then immersed in a polycation (or polyanion) solution to form the first positively (or negatively) charged layer. Negatively-charged enzymes are immobilized on the polycationic electrode through electrostatic forces. These deposition processes are carried out repeatedly to obtain the desired number of layers [2]. Poly(allyl amine), poly(L-lysine), poly(ethyleneimine), poly(dimethyl diallyl ammonium chloride), poly(allyl amine hydrochloride), and chitosan or its derivatives are the polycations commonly employed in layer-bylayer films deposition. The chitosan derivative has an excellent film-forming ability, high permeability toward water and small molecules, good adhesion, non-toxicity, high mechanical strength, and good biocompatibility. The polyanions most commonly 


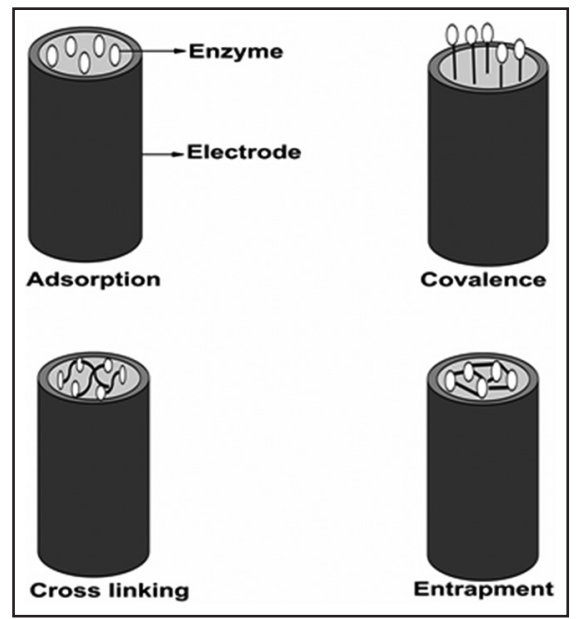

Figure 2: Immobilization schemes [2].

used are poly(styrene sulfonate), poly(vinyl sulfonate), poly(aniline propane sulfonic acid), poly(acrylic acid), and poly(methacrylic acid) [5]. The layer-by-layer technique provided a favorable microenvironment to keep the bioactivity of enzyme and to prevent enzyme leakage.

\subsubsection{Electrochemical doping}

Enzymes can also be immobilized by electrochemical doping. By controlling $\mathrm{pH}$, the negatively or positively charged enzymes can be doped into the conductive polymer film during its oxidation or reduction process, respectively. During oxidation, the polymer becomes positively charged. At a given $\mathrm{pH}$, negatively charged enzyme molecules can be incorporated into the conductive polymer during the oxidation process to form an enzyme electrode [6]

\subsection{Covalent Immobilization}

In this technique, the enzyme is covalently coupled to polymeric supports. For this purpose, biocatalysts are bound to the surface through their functional groups that are not essential for their catalytic activity. The binding of the enzymes to the solid support is generally carried out by initial activation of the surface using multifunctional reagents such as glutaraldehyde or carbodiimide followed by enzyme coupling to the activated support. The carrier support can either be an inorganic material, a natural (e.g., cellulose), or synthetic polymer (e.g., nylon). Covalent immobilization can be performed directly onto the transducer surface or onto a thin membrane fixed onto the transducer. Carbodiimides allow the binding between the carboxyl groups of a support and the amino function of an enzyme. N-hydroxysuccinimide can be associated to carbodiimide to improve immobilization efficiency. This procedure is widely used to develop enzymatic biosensors [2]. Carbodiimides also allow the binding between the amino groups of a support and the carboxyl function of enzyme (Figure 3).
Enzyme immobilization can also be achieved using glutaraldehyde as the activating agent. A first Schiffbase reaction occurs between an aldehyde group of glutaraldehyde and an amine function of the support. Then, the second aldehyde group of glutaraldehyde reacts with an amine function of enzyme (Figure 4).

\subsection{Entrapment}

\subsubsection{Electropolymerization}

Electropolymerization is an attractive approach for the controlled immobilization of enzymes on electrode surfaces. This one-step method consists of applying an appropriate potential or current to the transducer soaked in an aqueous solution containing both enzyme and monomer molecules. The immobilization of enzyme, mediators, and additives can be simultaneously achieved in the same sensing layer. The biological element is generally not modified, and the activity of the enzyme is preserved during the immobilization process. Variety of immobilization matrices such as polydimethylsiloxane, a photopolymer, a silica gel, a polysaccharide, or a carbon paste are used in fabrication. Biosensors based on physically entrapped enzymes are often suffered from leaching of biocomponent and possible diffusion barriers which restrict the performances of these systems [4].

\subsubsection{Entrapment in an amphiphilic network}

This technique exploits the swelling properties of the polymers such as solvent polarity. The indicator reagent and enzyme when added to solution around the polymer gradually diffuse from hydrophilic solution into the swollen hydrophilic phase of the polymer network. The indicator reagent and the enzyme can be entrapped in the hydrophilic and enzyme-friendly environment by drying [2].

\subsubsection{Sol-gel process}

Sol-gel process involves hydrolysis of alkoxide precursors under acidic (or alkaline) conditions followed by condensation of the hydroxylated units, which leads to the formation of a porous gel matrix in which an enzyme can be successfully entrapped. Silica gels are highly porous, showing physical rigidity, chemical and biological inertness, and thermal stability. These matrices often suffer from considerable shrinkage during the drying process, which leads to fracture of the material and pore collapse $[2,4]$.

\subsubsection{Entrapment in a polysaccharide-based gel}

Thepolysaccharide-based gelmatrices arebiocompatible, non-toxic, provide natural microenvironment to the enzyme, and also give sufficient accessibility to electrons to shuttle between the enzyme and the electrode. The enzymes can also be entrapped in a polysaccharidebased gel such as sodium alginate, chitosan, or agarose choline oxidase. Sodium alginate is a widely used monovalent salt as it has good gelification ability in the presence of divalent cations and the enzyme entrapment 


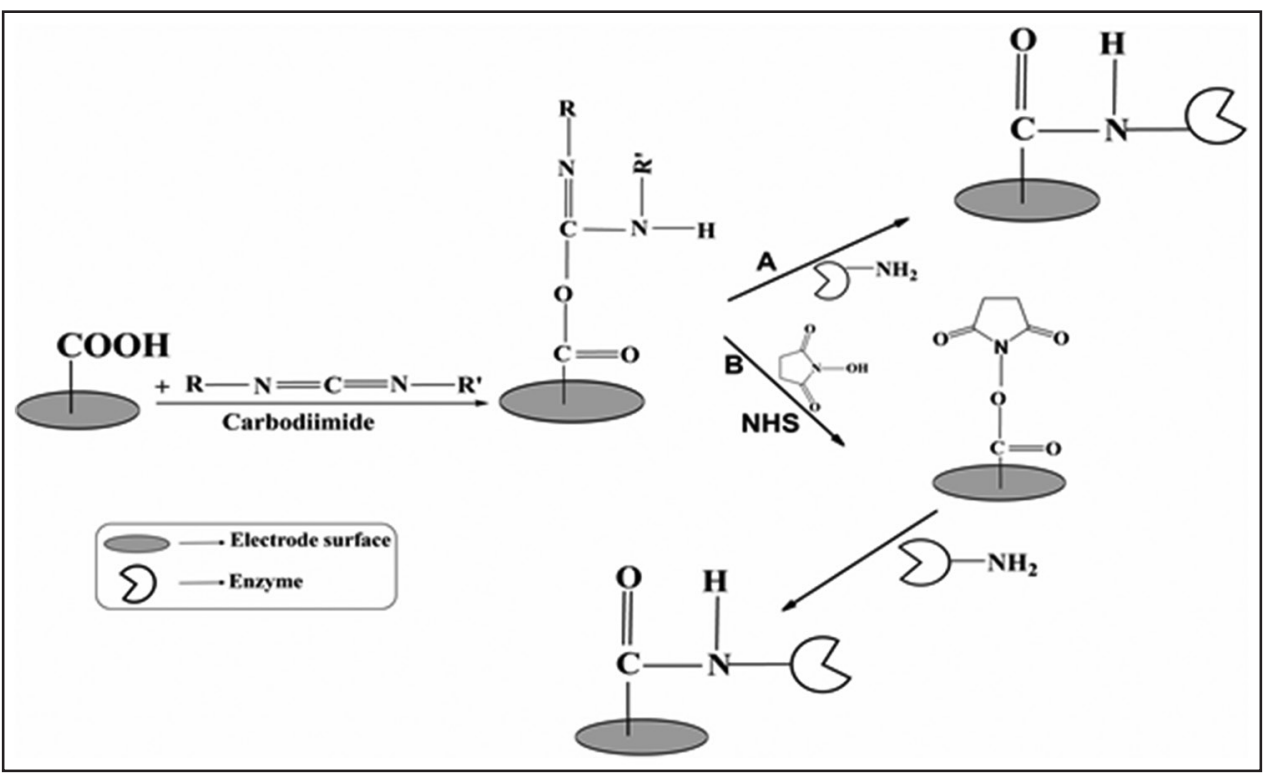

Figure 3: Immobilization of enzyme on carboxylated surface by carbodiimide coupling without (A) or with N-hydroxysuccinimide (B).

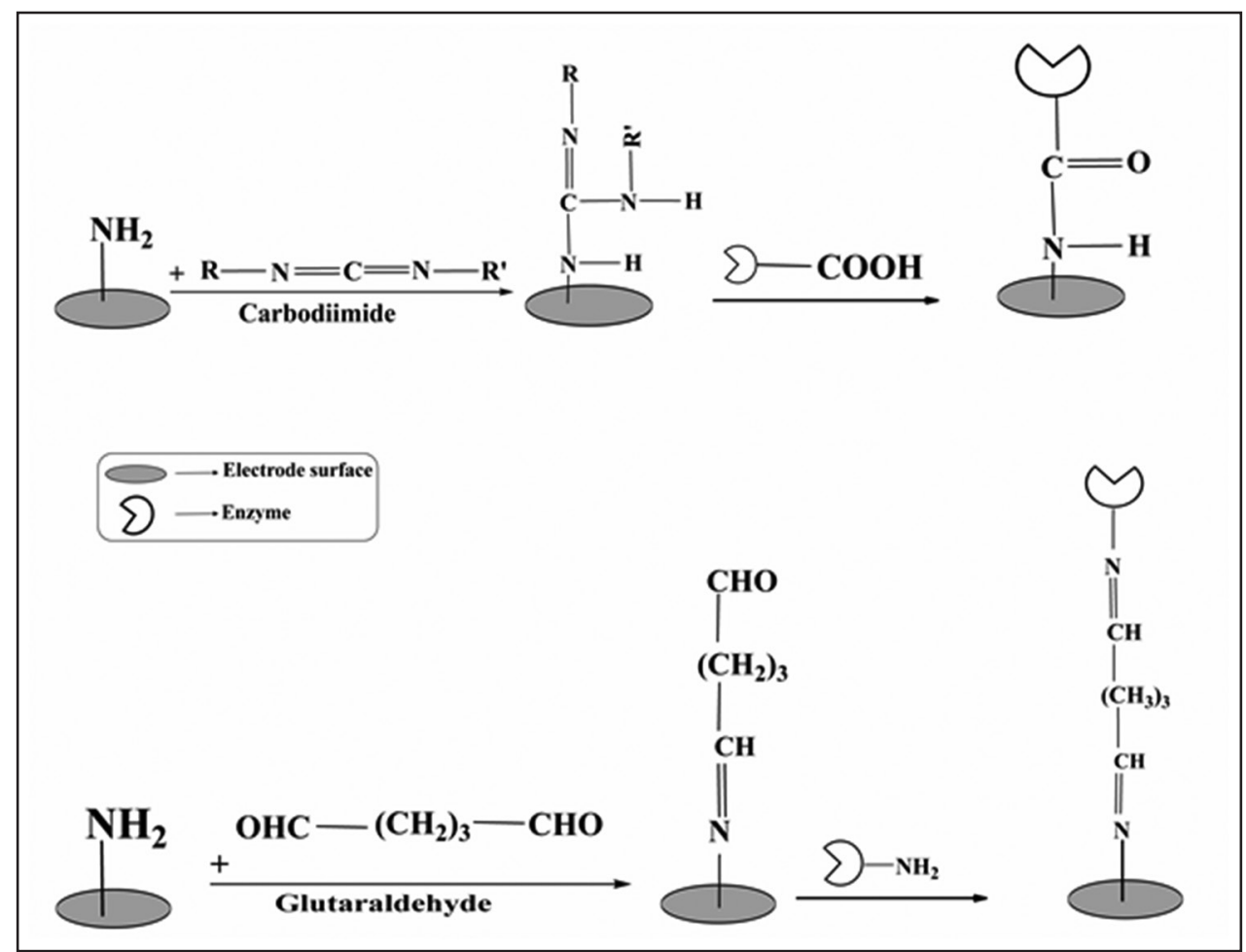

Figure 4: Immobilization of enzyme on laminated surface by carbodiimide and glutaraldehyde.

in an alginate gel is easy to achieve. However, gel is very porous, and its stability is poor and so it can cause enzyme leaching. Agarose powder can be dissolved in a buffer by heating and this mixture gellifies when temperature decreases. An enzyme can be entrapped by incorporating it in the mixture during gelling [2].

\subsubsection{Entrapment in a carbon paste}

Carbon paste is a mixture of carbon (graphite) powder, and it is used as a popular binder for the preparation of various electrodes, sensors, and detectors. Carbon paste electrodes modified with enzyme are prepared by first mixing enzyme solution and graphite powder. Then, the resulting enzymatic powder is mixed with mineral oils such as paraffin. The prepared paste is filled into a plastic cylindrical cartridge to get the sensor [7]. Carbon paste allows an intimate contact between incorporated enzymes, mediator, and sensing sites permitting a fast ET. It is versatile and stable, and the surface is easily renewed with good reproducibility. 


\subsection{Cross-linking}

Cross-linking is a popular method for the immobilization of enzymes by cross-linking with glutaraldehyde or other bifunctional agents such as glyoxal or hexamethylenediamine. The enzyme can be either cross-linked with each other or in the presence of a functionally inert protein such as bovine serum albumin. Covalent linking of biomolecules on the transducer is an efficient method of immobilization. The main drawback is the possibility of activity losses due to the distortion of the active enzyme [4].

\section{CONCLUSION}

The present study briefly surveys various enzyme immobilization methods employed in sensor development. The facts gathered in the study indicated that the immobilization is a key step in development of biosensor and the conducting polymers form an efficient class of materials which is highly suitable as transducers of sensor development. These materials not only show desired level of conductivity but also helpful in effective transduction and amplification of signals. The study revealed that the different immobilization methods such as physical adsorption, covalent linking, entrapment, and cross-linking are employed in sensor development. The study concludes that the choice of proper immobilization method and the conducting polymer greatly influence the performance of the developed sensor.

\section{REFERENCES}

1. M. Gerard, A. Chaubey, B. D. Malhotra, (2002) Application of conducting polymers to biosensors, Biosensors and Bioelectronics, 17: 345-359.

2. A. Sassolas, L. J. Blum, B. D. Leca-Bouvier, (2012) Immobilization strategies to develop enzymatic biosensors, Biotechnology Advances, 30: 489-511.

3. S. Nambiar, J. T. W. Yeow, (2011) Conductive polymer-based sensors for biomedical applications, Biosensors and Bioelectronics, 26: 1825-1832.

4. T. Ahuja, I. A. Mir, D. Kumara, R. Rajesh, (2007) Biomolecular immobilization on conducting polymers for biosensing applications, Biomaterials, 28: 791-805.

5. W. Zhao, J. J. Xu, H. Y. Chen, (2006) Electrochemical biosensors based on layer-bylayer assemblies, Electroanalysis, 18: 1737-1748.

6. J. Zhang, D. Shan, S. L. Mu, (2007) Improvement in selectivity and storage stability of a choline biosensor fabricated from poly(aniline-co-oaminophenol), Frontiers in Bioscience, 12: 783-790.

7. I. Svancara, K. Vytras, K. Kalcher, A. Walcarius, J. Wang, (2009) Carbon paste electrodes in facts, numbers, and notes: A review on the occasion of the 50-years jubilee of carbon paste in electrochemistry and electro analysis, Electroanalysis, 21: 7-28. 